\title{
Adaptation and validation of a questionnaire assessing patient satisfaction with pharmacy services in general hospitals
}

This article was published in the following Dove Press journal:

Patient Preference and Adherence

25 March 2014

Number of times this article has been viewed

\author{
Khalaf Ali Al-Jumah' \\ Mohamed Azmi Hassali \\ Ibrahem Al-Zaagi ${ }^{3}$
}

'Al Amal Psychiatric Hospital, Riyadh, Saudi Arabia; ${ }^{2}$ School of Pharmaceutical Sciences, Universiti Sains Malaysia, Penang, Malaysia; ${ }^{3}$ King Saud Medical City, Riyadh, Saudi Arabia
Correspondence: Khalaf Ali Al-Jumah Pharmacy Department, Al Amal Psychiatric Hospital, Riyadh, Saudi Arabia Email khalafaljumaah@yahoo.com
Objective: The aim of this study was to cross-culturally adapt the Armando Patient Satisfaction Questionnaire into Arabic and validate its use in the general population.

Methods: The translation was conducted based on the principles of the most widely used model in questionnaire translation, namely Brisling's back-translation model. A written authorization allowing translation into Arabic was obtained from the original author. The Arabic version of the questionnaire was distributed to 480 participants to evaluate construct validity. Statistical Package for Social Sciences version 17.0 for Windows was used for the statistical analysis.

Results: The response rate of this study was $96 \%$; most of the respondents (52.5\%) were female. Internal consistency was assessed using Cronbach's $\alpha$, which showed that this questionnaire provides a high reliability coefficient (reaching 0.9299 ) and a high degree of consistency and thus can be relied upon in future patient satisfaction research.

Keywords: cross-cultural, Arabic, survey

\section{Introduction}

The role of pharmacists in the health care system has grown in the last decade to encompass areas beyond the dispensing function; it has now extended to pharmaceutical care. ${ }^{1}$ These new roles are based on the concept of changing the pharmacist's focus from drug providing and distributing services to more patient-oriented approaches, including providing certain interventions after interpretation and assessment of prescription and providing information regarding usage, potential side effects, and how to manage drug-related problems. ${ }^{2,3}$ Pharmacists provide this care through patient assessment, disease management, continuing follow-up, and collaboration with other health care providers. ${ }^{2,3}$ The available literature regarding pharmacist services shows a positive impact of this care on patient outcome. ${ }^{4,5}$ In Saudi Arabia, health organizations took an official position towards patient oriented pharmacy practice when clinical pharmacy was adopted as a strategy in national drug use in the late 1970s. Since then, pharmacy practice in the hospital setting became more patient oriented. The concept of pharmaceutical care is now widely applied by pharmacists.

Obviously, there is a need to evaluate patient satisfaction with regard to these services to ensure continual improvement in pharmacist services. Service structure, processes, and outcomes are some examples of targets that can be used to evaluate the quality of pharmacist services. ${ }^{6,7}$ However, a search of the literature revealed that service outcome is not always included as a main criterion for evaluation. Pharmacist service evaluation criteria should not be restricted to evaluating only how well the services are delivered but should also focus on how well the services match patients' needs. Accordingly, 
patient satisfaction outcomes should be integrated into all future plans for evaluation of pharmacist services. ${ }^{7.8}$

The evidence shows that satisfaction positively affects the likelihood that patients will continue use of health care services and maintain a good relationship with health care providers. ${ }^{9,10}$ Moreover, patient adherence to medication has been reported to be significantly improved as a result of increased patient satisfaction., ${ }^{9,11,12}$ Patient satisfaction evaluation may help detect patients' needs, perceptions, and concerns and any shortcomings associated with the health care system. ${ }^{13}$ It is also an important indicator for stakeholders to continue monitoring and improving the quality of the health care system. ${ }^{13}$

However, it is difficult to determine patient satisfaction and identify a single factor that is directly associated with a low or high level of patient satisfaction. A variety of factors might be involved in the patient satisfaction process. These include the patient demographics and educational level. ${ }^{14}$ Furthermore, patient satisfaction level is directly associated with patient expectation. ${ }^{15,16}$ As such, patient satisfaction can be defined as the sum of the patient perceptions and expectations about the pharmacy service provided to them. ${ }^{17}$ If the perceived expectations are met, the patient will be satisfied. Otherwise, patients report a low satisfaction level when there is a clear discrepancy between their high expectations and a low perception of the services received. ${ }^{17}$

By performing a literature review, we found that there is no instrument available in Arabic that is specifically designed to evaluate patient satisfaction with pharmacy services. Therefore, the development and validation of such an instrument or translation and adaptation of a validated instrument is necessary to help researchers who are interested in exploring the quality of health care systems and patient satisfaction with services provided by pharmacists in Arabic countries.

The Armando Patient Satisfaction Questionnaire has been adapted and validated in various cultures and has been used in several countries in different settings for pharmacy services ${ }^{18,19}$ and is characterized by simplicity and practicality. Thus far, no cross-cultural adaptation of this questionnaire into Arabic has been performed. Therefore, the aim of this study is to crossculturally adapt and validate the Armando Patient Satisfaction Questionnaire into Arabic for use by the general population.

\section{Methods}

\section{Cultural adaptation}

The Patient Satisfaction Questionnaire has been validated and used in various cultures. ${ }^{18}$ The questionnaire consists of ten closed questions using a 5-point Likert scale from 1 (total disagreement) to 5 (strong agreement) and an open section to express comments. The evidence supports the translation of an existing questionnaire for the investigation of phenomena in situations where the language of the questionnaire is not the first language of the population under study.

A written authorization to translate the original English version of the patient satisfaction questionnaire into Arabic was obtained from Professor Pedro Armando at the Faculty of Chemical Sciences, National University of Córdoba.

The translation process was conducted based on the principles of the most widely used models in questionnaire translation, namely Brisling's back-translation model, which consists of four techniques: 1) back-translation, 2) bilingual technique, 3) committee approach, and 4) pre-test procedure. ${ }^{20}$

Two bilingual pharmacists, who are fluent in both Arabic and English, translated the patient satisfaction questionnaire into Arabic, and the content of the translated questionnaire was then reviewed by a group of researchers (committee approach) also fluent in Arabic and English. The goal was to convey the conceptual meaning of the original while rendering it culturally explicable for the Arabic context, rather than seeking linguistic equivalence.The work of Al-Muhtaseb and Mellish ${ }^{21}$ was followed to produce a natural Arabic text. Once the translation was completed, a professional translator and a health care professional reviewed it for clarity and for cultural appropriateness.

A pilot study with 20 patients was conducted using a testpretest technique to measure the validity of the Arabic version. The initial group reviewed the comments by members of the pilot sample and adapted the Arabic version accordingly.

A back-translation of the Arabic version to English was performed by a professional translator, with the original version of the questionnaire concealed. The back-translated and the original version of the questionnaire were compared by the original author, and subsequent discrepancies were resolved via email correspondence between the Arabic research team and the original author.

\section{Ethical consideration}

Ethical approval was obtained from the training and research committee in King Saud Medical City. Furthermore, the research protocol was approved by the ethics committee at the Ministry of Health.

\section{Participants and recruitment}

The study was conducted at King Saud Medical City in Riyadh, Saudi Arabia (1,400 beds) from August through September 2013.

Patients in the pharmacy's waiting area were invited randomly by a member of the research team to participate in the 
study. Informed written consent was obtained from patients who agreed to participate, and all subjects were able to read the content included in the study.

The sample size was calculated based on a minimum subject/item ratio of 20:1 $(20 \times 10=200) .{ }^{22,23}$ However, the Arabic version of the patient satisfaction questionnaire was distributed to a sample of 500 participants because it is known that increases in sample size tend to produce more accurate results. ${ }^{24}$ All patients who agreed to participate were informed that the results of the study would be kept confidential and anonymous and would not be viewed by health care providers, to reduce the risk of bias and to ensure a good relationship with health care providers.

Demographic data such as sex, age, level of education, and occupation were collected.

\section{Data analysis}

Statistical Package for Social Sciences version 17.0 for Windows (SPSS Inc., Chicago, IL, USA) was used for the statistical analysis. A descriptive analysis was performed on demographic variables by calculation of the means and standard deviations for each of the items related to satisfaction and the corresponding frequencies of each of the response scale points.

To evaluate the construct validity, a factor analysis was performed using principal component extraction and calculation of the Pearson correlation coefficient. To determine the validity of the Arabic version of the questionnaire, a correlation coefficient was calculated between each statement utilizing inter-item and item-total mean correlation.

\section{Results}

A total of 500 questionnaires were collected from patients; of these, 20 were excluded because of incompletion. A total of 252 participants, representing $52.5 \%$ of the total study sample, were female, while the remaining 228 (47.5\%) were male; participant ages ranged from 15 to 60 years. The majority of the participants $(52.8 \%)$ had a high school education level, $52.4 \%$ of the sample received three medications or more per visit from the ambulatory care pharmacy in King Saud Medical City in Riyadh, and 56.2\% received medications from the pharmacy three or more times per year. The final Arabic version of the patient satisfaction questionnaire consisted of three parts. The first part explained the aim of the study and the expected time it would take to complete the questionnaire. The second part concerned demographic characteristics of the participants (including age, sex, education level, occupation, annual number of pharmacy visits, and number of drugs dispensed per visit).
The third part contained ten questions using a 5-point Likert scale from 1 (total disagreement) to 5 (strong agreement). During pilot testing, patients did not report finding any of the statements unclear or having had any difficulty understanding the questionnaire, so the translated questionnaire was adapted without any modifications.

A correlation coefficient was calculated between each statement utilizing inter-item and item-total mean correlation using a Pearson correlation coefficient test; a positive correlation was shown between all statements and was statistically significant at $P \leq 0.01$, as shown in Table 1 .

Internal consistency was assessed using Cronbach's $\alpha$, which showed a high reliability coefficient (reaching 0.9299; Table 2) and a high degree of consistency and suitability for the application in future study.

From the results shown in Table 1, it is clear that the study sample is in good agreement on axis one ("When attending this pharmacy to acquire drugs ..."), with a range of 3.63-5.00. Similarly, the results from axis two ("As a consequence of the services received in this pharmacy ...") showed that the subjects were also in good agreement, on average, with scores ranging from 4.41 to 5.00 ("agree strongly").

\section{Discussion}

The aim of this study was to translate and adapt a patient satisfaction questionnaire into Arabic. The results show evidence of validity and reliability in evaluating patient satisfaction with dispensing services in a general hospital setting.

To the best of our knowledge, this is the first study describing cross-cultural adaptation in the Arabic version of the patient satisfaction questionnaire.

The results provide evidence for confirming reliability of the Arabic version of the patient satisfaction questionnaire. The Cronbach's $\alpha$ for axis one, axis two, and total scales $(0.8360,0.9122$, and 0.9299 , respectively) are comparable to those of the original questionnaire $(0.91)^{18}$ and the Spanish version (0.75). ${ }^{25}$ The inter-item correlations also tend to suggest that the items were measuring the same concept, ie, patient satisfaction, and are indicative of construct validity. ${ }^{26,27}$

One of the advantages of this study is the use of a sample from one of the largest general hospitals in Saudi Arabia for the validation of the Arabic version of a patient satisfaction questionnaire. This sample may overcome any cultural and environmental factors that could lead to differences in the instrument's ability to measure an object of interest. The high response rate to the questionnaire can be explained 
Table I Responses of the study sample to questionnaire statements (in descending order according to the average percentage agreeing)

\begin{tabular}{|c|c|c|c|c|c|c|c|c|}
\hline Statement & $\begin{array}{l}\text { Totally } \\
\text { disagree }\end{array}$ & Disagree & $\begin{array}{l}\text { Either agree } \\
\text { or disagree }\end{array}$ & Agree & $\begin{array}{l}\text { Strongly } \\
\text { agree }\end{array}$ & Mean & SD & $\begin{array}{l}\text { Correlation } \\
\text { coefficient } \\
\text { with axis }\end{array}$ \\
\hline \multicolumn{9}{|c|}{ When attending this pharmacy to acquire drugs } \\
\hline $\begin{array}{l}\text { I. I received adequate information } \\
\text { about how I should use my drugs }\end{array}$ & $25(5.2 \%)$ & $33(6.9 \%)$ & $58(12.1 \%)$ & $281(58.4 \%)$ & $84(17.5 \%)$ & 3.76 & 0.996 & $0.879 * *$ \\
\hline $\begin{array}{l}\text { 2. The staff appear interested in } \\
\text { helping me with the use of my } \\
\text { drugs }\end{array}$ & $33(6.9 \%)$ & $35(7.3 \%)$ & $69(14.3 \%)$ & $264(54.9 \%)$ & $80(16.6 \%)$ & 3.67 & 1.057 & $0.846 * *$ \\
\hline 3. Staff were diligent during my visit & $45(9.4 \%)$ & $63(13.1 \%)$ & $74(15.4 \%)$ & $223(46.4 \%)$ & $76(15.8 \%)$ & 3.46 & 1.176 & $0.882 * *$ \\
\hline \multicolumn{9}{|c|}{ As a consequence of the service received in this pharmacy } \\
\hline $\begin{array}{l}\text { 4. I learnt about the need to carry } \\
\text { out the treatment prescribed by } \\
\text { my doctors }\end{array}$ & $27(5.6 \%)$ & $37(7.7 \%)$ & $95(19.8 \%)$ & $25 \mathrm{I}(52.2 \%)$ & $7 \mathrm{I}(14.8 \%)$ & 3.63 & 1.011 & $0.780 * *$ \\
\hline $\begin{array}{l}\text { 5. I would keep the staff of this } \\
\text { pharmacy informed about any } \\
\text { change in my drugs }\end{array}$ & $34(7.1 \%)$ & $31(6.4 \%)$ & $107(22.2 \%)$ & $238(49.5 \%)$ & $71(14.8 \%)$ & 3.58 & 1.046 & $0.785^{* *}$ \\
\hline $\begin{array}{l}\text { 6. I improved my knowledge about } \\
\text { the drugs I use }\end{array}$ & $26(5.4 \%)$ & $45(9.4 \%)$ & $100(20.8 \%)$ & $255(53.0 \%)$ & 55 (I I.4\%) & 3.56 & 0.994 & $0.782 * *$ \\
\hline $\begin{array}{l}\text { 7. I am satisfied with the service } \\
\text { received }\end{array}$ & 63 (13.1\%) & 55 (I I.4\%) & 76 (15.8\%) & 198 (4I.2\%) & 89 (18.5\%) & 3.41 & 1.276 & $0.878 * *$ \\
\hline $\begin{array}{l}\text { 8. I would recommend my friends } \\
\text { and family to attend this pharmacy } \\
\text { to receive its service }\end{array}$ & 53 (। I\%) & $6 \mathrm{I}(12.7 \%)$ & 127 (26.4\%) & 183 (38.0\%) & 57 (I I.9\%) & 3.27 & 1.163 & $0.868 * *$ \\
\hline $\begin{array}{l}\text { 9. I would keep attending } \\
\text { this pharmacy which has given me } \\
\text { advice about my drugs }\end{array}$ & 48 (10\%) & $7 \mid(\mid 4.8 \%)$ & $129(26.4 \%)$ & 173 (36.0\%) & 60 (12.5\%) & 3.26 & I.I57 & $0.838 * *$ \\
\hline $\begin{array}{l}\text { 10. I learnt to understand about the } \\
\text { side effects of the drugs I use }\end{array}$ & $60(12.5 \%)$ & 82 (I7\%) & I I 8 (24.5\%) & 168 (34.9\%) & 53 (I I.0\%) & 3.15 & 1.200 & $0.878^{* *}$ \\
\hline
\end{tabular}

Note: $* * P \leq 0.01$.

Abbreviation: SD, standard deviation.

by its design, which takes into consideration the deficiencies observed in other questionnaires, such as a large number of questions, including all the activities performed by dispensing pharmacists, which may discourage participants from completing the questionnaire.

One of limitations of this study was that certain differences between the two languages made translation and back-translation difficult. The general structure of the English sentence is subject + verb + object, which differs from the Arabic verb + subject + object. ${ }^{21}$

This questionnaire showed good internal consistency and a component structure identical to the original English

Table 2 Cronbach's $\alpha$

\begin{tabular}{llc}
\hline Axes & $\begin{array}{l}\text { Number of } \\
\text { statements }\end{array}$ & Stability \\
\hline $\begin{array}{l}\text { When attending this pharmacy } \\
\text { to acquire drugs }\end{array}$ & 3 & 0.8360 \\
$\begin{array}{l}\text { As a consequence of the service } \\
\text { received in this pharmacy }\end{array}$ & 7 & 0.9122 \\
General reliability coefficient & 10 & 0.9299 \\
\hline
\end{tabular}

version. It will be a suitable instrument to assist patient satisfaction in Arabic speaking countries, and will help pharmacists in Saudi Arabia to predict the quality and availability of pharmaceutical care services in their practice.

Further work to investigate patient satisfaction with drug dispensing in different settings is necessary.

\section{Conclusion}

We cross-culturally adapted an Arabic version of the Armando Patient Satisfaction Questionnaire for use among the general population. The Arabic version presented good internal consistency and a component structure identical to the original English version.

\section{Disclosure}

The authors report no conflicts of interest in this work.

\section{References}

1. Hepler CD, Strand LM. Opportunities and responsibilities in pharmaceutical care. Am J Hosp Pharm. 1990;47(3):533-543.

2. Beney J, Bero L, Bond CM. Expanding the roles of outpatient pharmacists: effects on health services utilisation, costs, and patient outcomes. Cochrane Database Syst Rev. 2000;(3):CD000336. 
3. Nkansah N, Mostovetsky O, Yu C, et al. Effect of outpatient pharmacists' non-dispensing roles on patient outcomes and prescribing patterns. Cochrane Database Syst Rev. 2010;(7):CD000336.

4. Armour C, Bosnic-Anticevich S, Brillant M, et al. Pharmacy Asthma Care Program (PACP) improves outcomes for patients in the community. Thorax. 2007;62(6):496-592.

5. Crockett JA, Taylor SJ, McLeod LJ. Patient responses to an integrated service, initiated by community pharmacists, for the prevention of osteoporosis. Int J Pharm Pract. 2008;16(2):65-72.

6. Kozma C, Reeder C, Schulz R. Economic, clinical, and humanistic outcomes: a planning model for pharmacoeconomic research. Clin Ther. 1992;15(6):1121-1132; discussion 1120.

7. Johnson JA, Coons SJ, Hays RD, Sabers D, Jones P, Langley PC. A comparison of satisfaction with mail versus traditional pharmacy services. J Manag Care Pharm. 1997;3:327-337.

8. Panvelkar PN, Saini B, Armour C. Measurement of patient satisfaction with community pharmacy services: a review. Pharm World Sci. 2009;31(5):525-537.

9. Dang BN, Westbrook RA, Black WC, Rodriguez-Barradas MC, Giordano TP. Examining the link between patient satisfaction and adherence to HIV care: a structural equation model. PloS One. 2013;8(1):e54729.

10. Dubina MI, O'Neill JL, Feldman SR. Effect of patient satisfaction on outcomes of care. Expert Rev Pharmacoecon Outcomes Res. 2009;9(5): 393-395.

11. Locker D, Dunt D. Theoretical and methodological issues in sociological studies of consumer satisfaction with medical care. Soc Sci Med Med Psychol Med Sociol. 1978;12:283-292.

12. Schommer JC, Kucukarslan SN. Measuring patient satisfaction with pharmaceutical services. Am J Health Syst Pharm. 1997;54(23): 2721-2732.

13. Ford RC, Bach SA, Fottler MD. Methods of measuring patient satisfaction in health care organizations. Health Care Manag Rev. 1997;22(2):74-89.

14. Alturki M, Khan TM. A study investigating the level of satisfaction with the health services provided by the Pharmacist at ENT hospital, Eastern Region Alahsah, Kingdom of Saudi Arabia. Saudi Pharm J. 2013;21(3):255-260.
15. Mira JJ, Aranaz J. La satisfacción del paciente como una medida del resultado de la atención sanitaria [Patient satisfaction as an outcome measure in health care]. Med Clin (Barc). 2000;114:26-33. Spanish.

16. Hall JA, Dornan MC. Meta-analysis of satisfaction with medical care: description of research domain and analysis of overall satisfaction levels. Soc Sci Med. 1988;27(6):637-644.

17. Ikegami N, Kawakita H. Patient satisfaction and hospital management policy. Jpn Hosp. 1987;6:11-15.

18. Armando PD, Pérez SRM, Pallarés MM, Uthurry NHS, Dáder MJF. Development and validation of a Spanish language patient satisfaction questionnaire with drug dispensing. Pharm World Sci. 2008;30(2):169-174.

19. Armando PD, Martínez Pérez SR, Molina Guerra AC, Martí Pallarés M, Solá Uthurry NH, Faus Dáder MJ. [Development and validation of a patient satisfaction questionnaire on pharmaceutical care in community pharmacies]. Rev Calid Asist. 2011;27(6):311-318. Spanish.

20. Brislin RW. Back-translation for cross-cultural research. J Cross Cult Psychol. 1970;1(3):185-216.

21. Al-Muhtaseb H, Mellish C, editors. Some Differences between Arabic and English: A Step Towards an Arabic Upper Model. 6th International Conference on Multilingual Computing; 1998; Cambridge, England.

22. Comrey AL, Lee HB. A First Course in Factor Analysis. London: Routledge; 1992.

23. Osborne JW, Costello AB. Sample size and subject to item ratio in principal components analysis. Pract Assess Res Eval. 2004; 9(11):8.

24. de Winter J, Dodou D, Wieringa P. Exploratory factor analysis with small sample sizes. Multivariate Behav Res. 2009;44(2):147-181.

25. Armando P, Martínez PS, Molina GA, Martí PM, Solá UN, Faus DM [Development and validation of a patient satisfaction questionnaire on pharmaceutical care in community pharmacies]. Rev Calid Asist. 2012;27(6):311-318. Spanish.

26. Ware JE, Wright W, Snyder MK, Chu G. Consumer perceptions of health care services: implications for the academic medical community. J Med Educ. 1975;50(9):839-848.

27. Thompson B, Daniel LG. Factor analytic evidence for the construct validity of scores: a historical overview and some guidelines. Educ Psychol Meas. 1996;56(2):197-208.
Patient Preference and Adherence

\section{Publish your work in this journal}

Patient Preference and Adherence is an international, peer-reviewed, open access journal focusing on the growing importance of patient preference and adherence throughout the therapeutic continuum. Patient satisfaction, acceptability, quality of life, compliance, persistence and their role in developing new therapeutic modalities and compounds to

\section{Dovepress}

optimize clinical outcomes for existing disease states are major areas of interest. This journal has been accepted for indexing on PubMed Central. The manuscript management system is completely online and includes a very quick and fair peer-review system. Visit http://www.dovepress.com/ testimonials.php to read real quotes from published authors. 